\title{
In-Situ Strain Monitoring in Deltoid of Composite T-joints using Optical Fiber
}

\author{
Shinsaku Hisada ${ }^{1, a}$, Shu Minakuchi ${ }^{1, b}$ and Nobuo Takeda ${ }^{1, \mathrm{c},{ }^{*}}$ \\ ${ }^{1}$ Department of Advanced Energy, Graduate School of Frontier Sciences, The University of \\ Tokyo, 5-1-5 Kashiwanoha, Kashiwa-shi, Chiba 277-8561, Japan \\ ahisada@smart.k.u-tokyo.ac.jp, bminakuchi@smart.k.u-tokyo.ac.jp, \\ takeda@smart.k.u-tokyo.ac.jp
}

\section{Keywords: Optical Fiber, T-joint, Deltoid, Process Monitoring}

\begin{abstract}
Composite $\mathrm{T}$-joints have a critical problem that cracks occur in the deltoid during curing because of the cure stress and thermal stress. There are few studies on the behavior of Tjoints during curing, and what is actually occurring in the deltoid is not well understood. In the present study, optical fiber was embedded in the deltoid using a new technique to clarify the internal state of the deltoid during curing. The embedded optical fiber successfully measured the process-induced strain distribution in the deltoid. Based on the experimental results and finite element analysis, a failure index for a process-induced failure of deltoid was discussed. An energy-based failure index could precisely predict the process-induced failure.

\section{Introduction}

One of the difficulties in composite structural application is joining components. A T-joint is a typical joining component of aircraft structures and composed of two L-shaped parts, a skin laminate, and a deltoid, which is filling a gap between other components (Fig. 1). In T-joints, it is reported that cracks occur in the deltoid during curing because of the cure stress and thermal stress [1]. This is a critical problem because such cracking significantly reduces the mechanical performance of T-joints. Although many studies on the behavior of T-joints under mechanical loadings have been conducted, studies on the behavior of these joints during curing are very limited, and it is not well understood what is actually occurring inside the deltoid. This is because, to date, there was no technology to evaluate the stress-strain state of the deltoid surrounded by other components during curing. In the present study, strain distribution inside the deltoid is measured using a new technique to embed an optical fiber into the deltoid, and the stress-strain state of the deltoid during curing is analyzed. Based on the experimental results, finite element analysis is conducted and a failure index for a process-induced failure of deltoid is discussed.
\end{abstract}




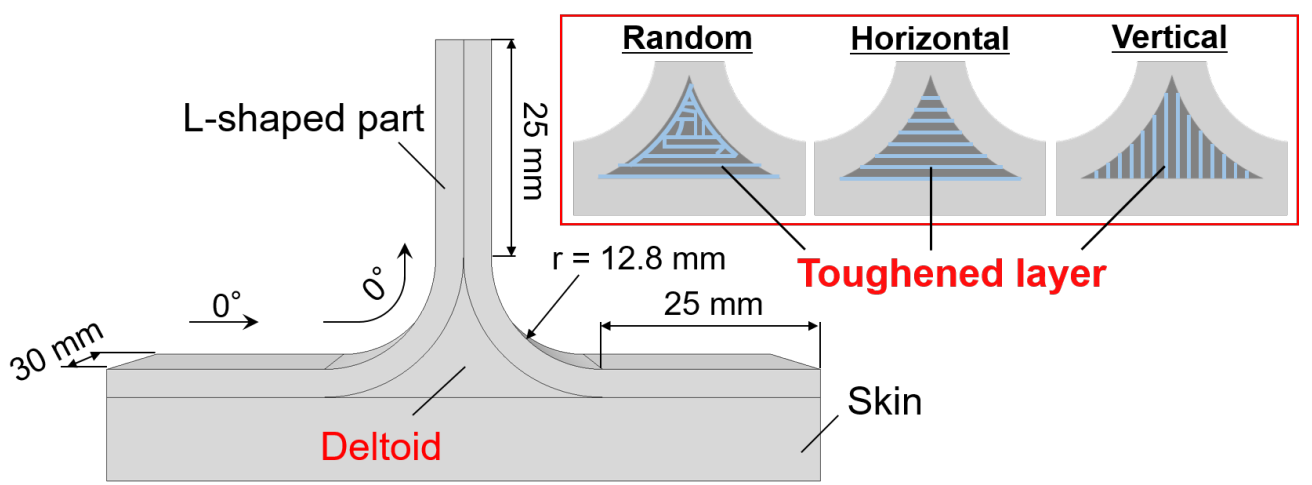

Fig. 1 Schematic of T-joint specimen used for in-situ strain measurement.

\section{In-Situ Strain Measurement of the Deltoid during Curing}

Distributed-Strain Measurement using an Optical Fiber. Optical fibers are widely used for strain measurement of composite materials because of various advantages, such as their small diameter and light weight [2]. In this study, strain distribution inside the deltoid was measured using an optical distributed sensor interrogator (ODiSI, LUNA Innovations, Inc.). The ODiSI uses swept-wavelength coherent interferometry [3]. The measurement principle is shown in Fig. 2. One of the split light paths is directly incident on the detector, whereas the other light paths is incident on the optical fiber and causing Rayleigh scattering. The location of the Rayleigh scattering can be determined from the time difference of the two lights. The frequency $v$ of the Rayleigh-scattered light shifts linearly with temperature change $(\Delta T)$ and axial strain change $(\Delta \varepsilon)$; this can be expressed as

$$
\frac{-\Delta v}{v}=K_{T} \Delta T+K_{\varepsilon} \Delta \varepsilon
$$

where $K_{T}$ and $K_{\varepsilon}$ are the coefficients. Therefore, the strain can be calculated from the Rayleigh-scattered light frequency by compensating the temperature effect.

\section{Materials and Methods}

A schematic of the specimen is shown in Fig. 1. A unidirectional carbon/epoxy prepreg sheet (T800S/3900-2B; Toray Industries, Inc.) was used in this study. This prepreg has been implemented in primary composite structures of Boeing 787, and has an interlaminar toughened layer to improve the interlaminar fracture toughness and impact resistance. This layer is composed of thermoplastic particles that are dispersed across the prepreg surface. The stacking sequence was $\left[\mathrm{O}_{2} / 90_{2}\right]_{2 \mathrm{~S}}$ (thickness: $3.2 \mathrm{~mm}$ ) in the L-shaped parts, and [02/902 $]_{23}$ (thickness: 9.6 $\mathrm{mm}$ ) in the skin laminate. The carbon fibers were oriented in the $90^{\circ}$ direction. Since it has been reported that materials with interlaminar toughened layers possess different transverse properties in the parallel direction and in the orthogonal direction to the toughened layer [4], three types of deltoid structures with differently oriented interlaminar toughened layers were prepared to investigate the effect of the toughened layer on the process-induced failure as shown in Fig. 1. An optical fiber (coating diameter $\phi=150 \mu \mathrm{m}$ ) was embedded along the centerline of the deltoid in a minimally invasive manner through tiny holes that were machined in the aluminum tool, deltoid, and skin. The holes in the deltoid and skin were made by pressing a thin needle $(\phi=0.5$ $\mathrm{mm}$ ) into the stacked prepreg sheets using an ultrasonic horn and a jig to adjust the pressing 
angle. By using this method, the strain in a closed structure surrounded by tools can be measured (Fig. 3). A K-type thermocouple was also embedded to compensate the effect of the temperature change. The specimens were cured in the autoclave at $180^{\circ} \mathrm{C}$ under $0.6 \mathrm{MPa}$ pressurization. The Rayleigh-scattered light frequency was measured at $2 \mathrm{~mm}$ intervals in the random deltoid and horizontally-aligned deltoid specimens, and at $4 \mathrm{~mm}$ intervals in the vertically aligned deltoid specimen.

Results. Figure 4 shows the local strain development during curing at the bottom of the random deltoid specimen. After gelation, the chemical cure shrinkage was measured as the cure reaction

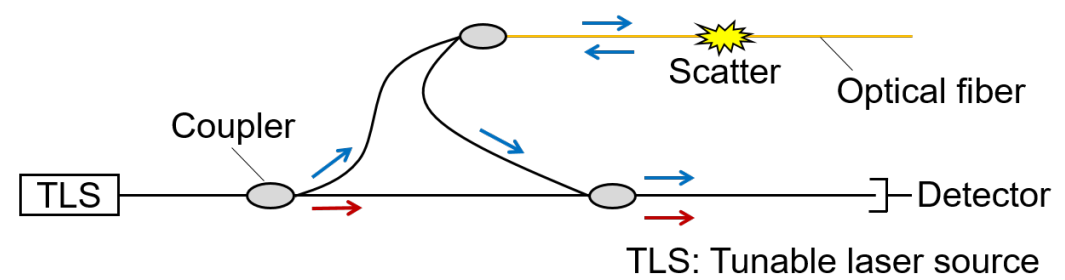

Fig. 2 Distributed-strain measurement principle using an ODiSi.

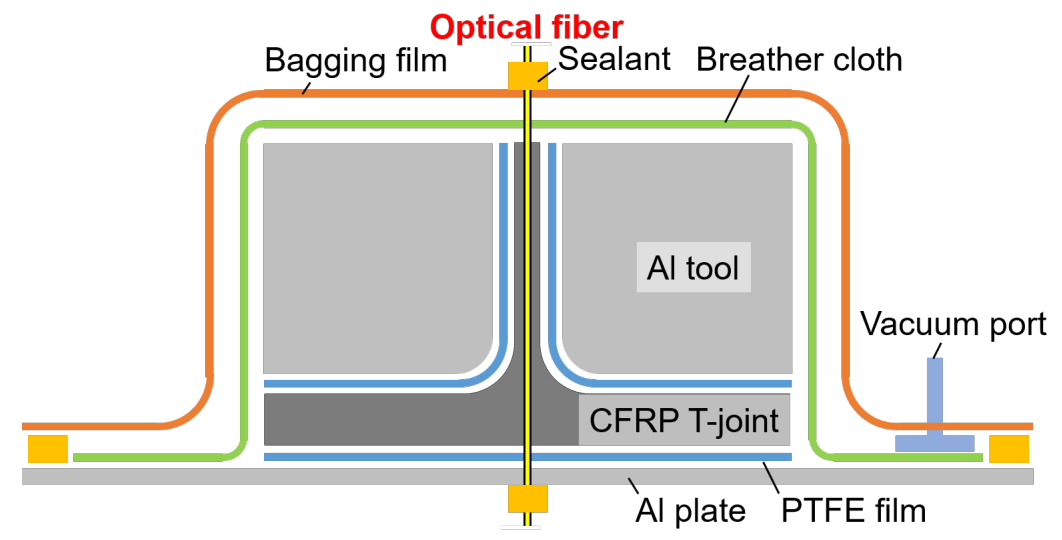

Fig. 3 Assembly and bagging of specimen with optical fiber.

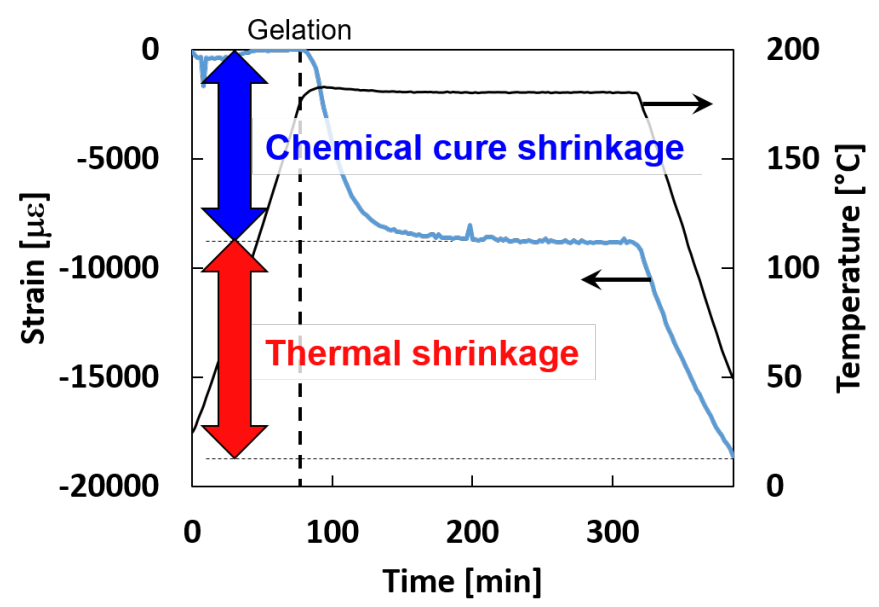

Fig. 4 Local strain development during curing at the bottom of the random deltoid specimen. 
proceeded. In addition, the thermal shrinkage was measured during cooling process. Therefore, it was clearly demonstrated that the strain development inside the deltoid can be measured by using the proposed technique. The comparison of the strain distribution in the deltoid at the room temperature for each specimen is shown in Fig. 5. The horizontal and vertical axes represent the amount of strain and the position along the optical fiber path, respectively, with $0 \mathrm{~mm}$ corresponding the top of the deltoid, and $16 \mathrm{~mm}$ corresponding to the bottom of the deltoid. In all specimens, small strain was measured in the upper region of the deltoid because this region was strongly constrained by the fiber layers of L-shaped parts. The strain increased toward the lower part of the deltoid as the constraint in the axial direction of the optical fiber weakened. Although similar trends of the strain distribution were measured in all specimens, the strain amount was the largest in the horizontally aligned deltoid specimen, and the smallest in the vertically aligned deltoid specimen. This is because larger strain occurs in the direction orthogonal to the toughened layers than in the direction that is parallel to the toughened layer direction as has been demonstrated in previous research [4].

Because failure did not occur in the specimens that were cured under the test conditions of this study, the specimens were cooled at approximately $6^{\circ} \mathrm{C} / \mathrm{min}$ using liquid nitrogen. Deltoid failure occurred at $-115^{\circ} \mathrm{C}$ for the random deltoid specimen, $-40{ }^{\circ} \mathrm{C}$ for the horizontally aligned specimen, and $-25^{\circ} \mathrm{C}$ for the vertically aligned deltoid specimen. A lateral crack forming in the upper region of the deltoid was observed in the random deltoid specimen and the horizontally aligned deltoid

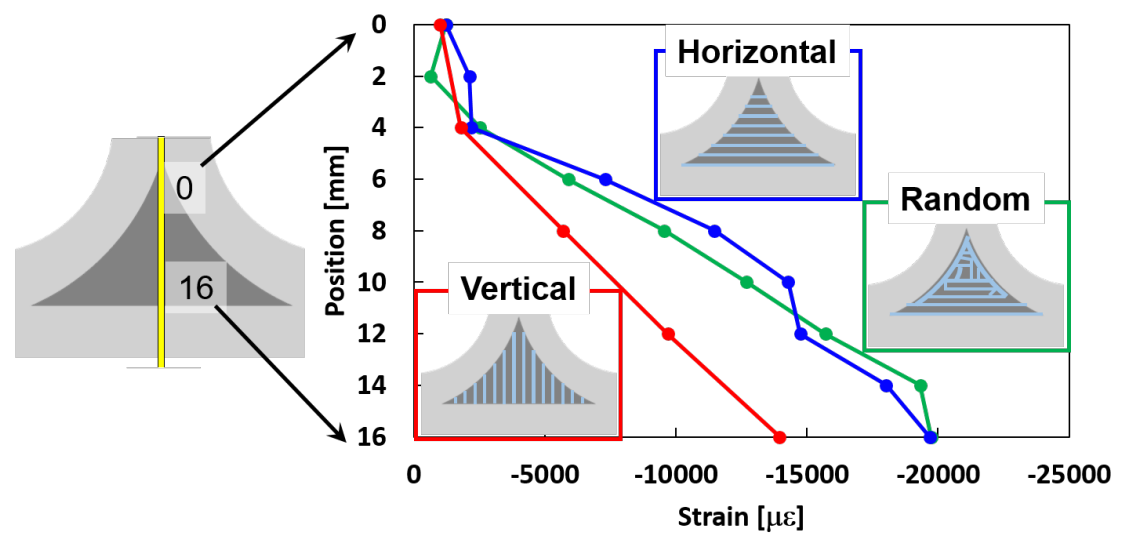

Fig. 5 Comparison of process-induced strain distribution in the deltoid for the three types of specimens.

(a)

(b)

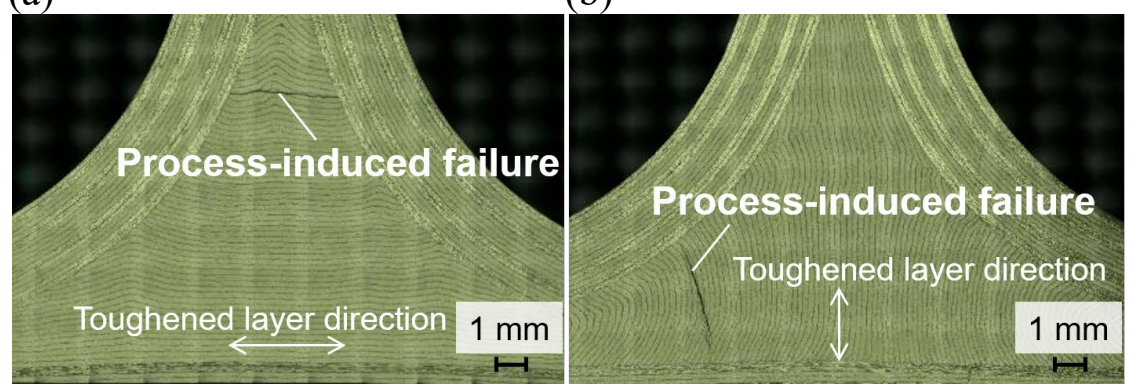

Fig. 6 Micrograph of failure surface.

(a) Horizontally aligned deltoid specimen. (b) Vertically aligned deltoid specimen. 
specimen, and a vertical crack forming in the lower region of the deltoid was observed in the vertically aligned deltoid specimen (Fig. 6).

\section{Discussion on the Process-Induced Failure Index using Finite Element Analysis Supported by Strain Measurement}

FEA Methods. FEA was performed using commercial software Abaqus 2017 (Dassault Systems, Inc.). Considering the symmetry of the $\mathrm{T}$-joint, only a quarter of the $\mathrm{T}$-joint was modeled, and symmetric boundary conditions were set for each plane. The horizontally aligned deltoid specimen was modeled, and the chemical cure shrinkage strain and thermal shrinkage strain associated with temperatures ranging from the curing temperature $\left(180{ }^{\circ} \mathrm{C}\right)$ to the failure temperature $\left(-40^{\circ} \mathrm{C}\right)$ were implemented. In the analysis of the curing stress, an incrementally linear elastic (ILE) model [5] was used to account for a modulus development during curing. In the ILE model, the process of chemical cure shrinkage was divided into several steps, and the corresponding shrinkage strain was implemented as the resin modulus changed after each step. The values of the shrinkage strain and the resin modulus at each step were estimated in the previous study of our research group [4] using two optical fibers. This technique is described in detail in [6]. The material properties of the fiber/resin layer were derived from the estimated resin modulus and carbon fiber properties using the self-consistent field micromechanics model [5]. Furthermore, the material properties of the entire material, including the interlaminar toughened layers, were calculated based on the rule of mixture. Material properties used in the analysis are described in Tables 1-2 [4, 7, 8]. The total curing stress was calculated by adding the stress generated in each step. A user subroutine UMAT of Abaqus was used for the material properties calculations and ILE model calculations.

Table 1. Resin modulus development and corresponding strain values associated with chemical cure shrinkage [4].

\begin{tabular}{ccccccccc}
\hline Step & 1 & 2 & 3 & 4 & 5 & 6 & 7 & 8 \\
\hline$E_{\mathrm{m}}[\mathrm{MPa}]$ & 8.10 & 23.5 & 41.6 & 60.0 & 83.0 & 117 & 158 & 167 \\
\hline$\Delta \varepsilon[\mu \varepsilon]$ & -960 & -920 & -690 & -520 & -380 & -270 & -270 & -290 \\
\hline
\end{tabular}

Table 2. Properties of materials used in this study [7, 8].

\begin{tabular}{cccccc}
\hline & & Carbon fiber & $\begin{array}{c}\text { Interlaminar } \\
\text { toughened layer }\end{array}$ & $\begin{array}{c}\text { Optical fiber } \\
\text { (Cladding, core) }\end{array}$ & $\begin{array}{c}\text { Optical fiber } \\
\text { (Coating) }\end{array}$ \\
\hline Elastic moduli & $E_{11}$ & 294 & 4.6 & 73.1 & 1.5 \\
[GPa] & $E_{22}$ & 19.5 & & & \\
& $G_{12}$ & 27.0 & & & \\
Poisson's ratio & $G_{23}$ & 27.0 & 0.44 & 0.16 & 0.25 \\
& $v_{12}$ & 0.17 & & & \\
\hline
\end{tabular}




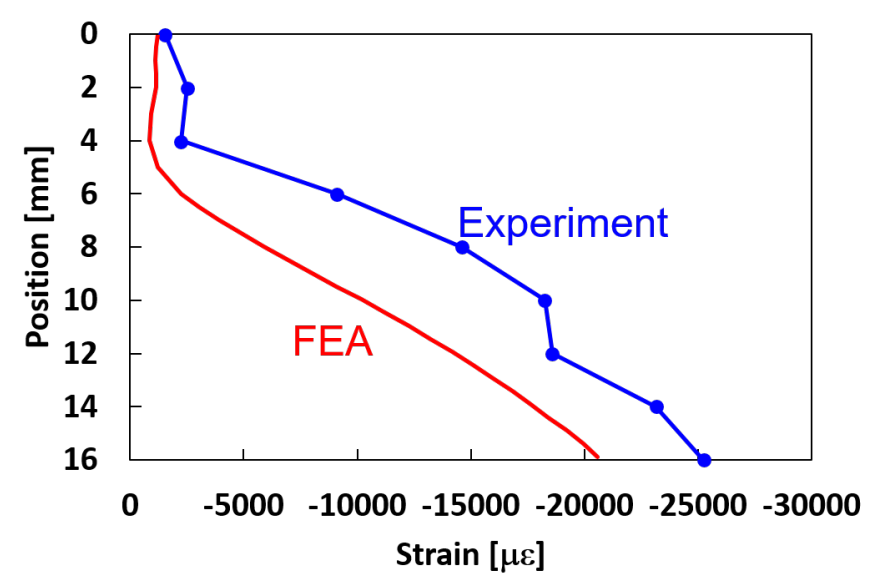

Fig. 7 Comparison of experimental and simulated strain distribution in the horizontally aligned deltoid specimen.

Results. Figure 7 shows a comparison of the experimental and simulated strain distribution in the deltoid of the horizontally aligned deltoid specimen. The shapes of the strain distribution curves were similar, and the amount of strain measured in the experiment was slightly larger than the simulated amount of strain. This is likely because actual deltoids are less constrained than their modeled counterparts because of imperfections such as misalignment of the fiber orientation. This result confirmed the validity of the simulated result, and the importance of acquiring in-situ strain using an optical fiber. Figure 8 shows the comparison of the normal stress state in the direction to the upper corner of the deltoid and the actual failure location. Although the maximum stress was generated near the corner of the deltoid, process-induced failure occurred at a location that was slightly distanced from the corner.

Discussion on the Process-Induced Failure Index. The FEA result implies that the stress-based failure index cannot be used to predict the location of the process-induced failure of deltoids. Therefore, the use of an energy-based failure index [9, 10] was investigated as a more precise index. The energy release rate can be expressed as the amount of work divided by the crack area.

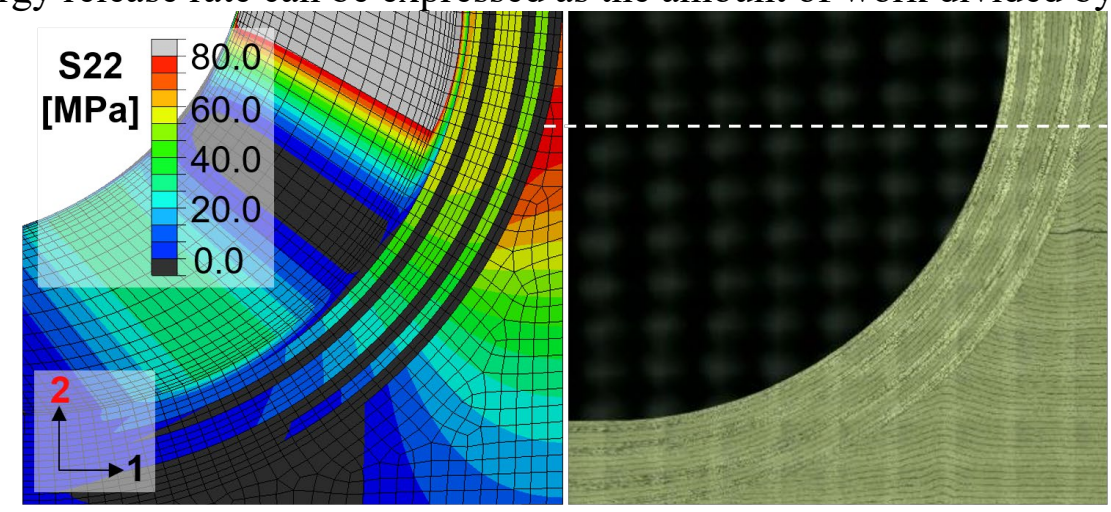

Fig. 8 Comparison of the simulated maximum stress location and actual failure location in the horizontally aligned deltoid speimen.

Therefore, when a crack with a unit length occurs in the $90^{\circ}$ layer of the cross-ply laminate under the constant-stress condition, the energy release rate is expressed as follows: 


$$
G=G_{C}=\frac{\delta W_{0}}{t}
$$

where $G c$ is the fracture toughness of the material, $\delta W_{0}$ is the amount of work required for crack initiation (Fig. 9), and $t$ is the thickness of the specimen. When the laminate is divided into similar $n \times n$ laminates, the stiffness values before and after crack initiation are the same as that for the original laminate. Then, the energy release rate can be expressed as follows:

$$
G=G_{C}=\frac{\delta W}{\left(\frac{1}{n} \times n^{2}\right) t}=\frac{\delta W}{n t}
$$

where $\delta \mathrm{W}$ is the amount of work associated with the generation of all cracks. Because the fracture toughness of the material is constant,

$$
\delta W=n \delta W_{0} .
$$

According to the similarity law of a triangle, $\sqrt{n}$ times stress is required to obtain $n$ times the work (Fig. 9). More specifically, when the width of the $90^{\circ}$ layer is $m$ times, $1 / \sqrt{m}$ times stress is required for crack initiation. At the deltoid, the $x$ axis and width of the deltoid $a(x)$ are acquired as shown in Fig. 10, and the normal stress required for crack initiation at the width of $a(x)$ is $\sigma(x)$. If the normal stress required for crack initiation at the unit width is $\sigma_{0}$, then

$$
\sigma(x)=\frac{1}{\sqrt{a(x)}} \sigma_{0}
$$

where

$$
\sqrt{a(x)} \sigma(x)=\sigma_{0}=\text { const }
$$

Because the $\sqrt{a(x)} \sigma(x)$ value required for crack initiation at a certain location in the deltoid is constant, a crack occurs at the maximum point of this value.

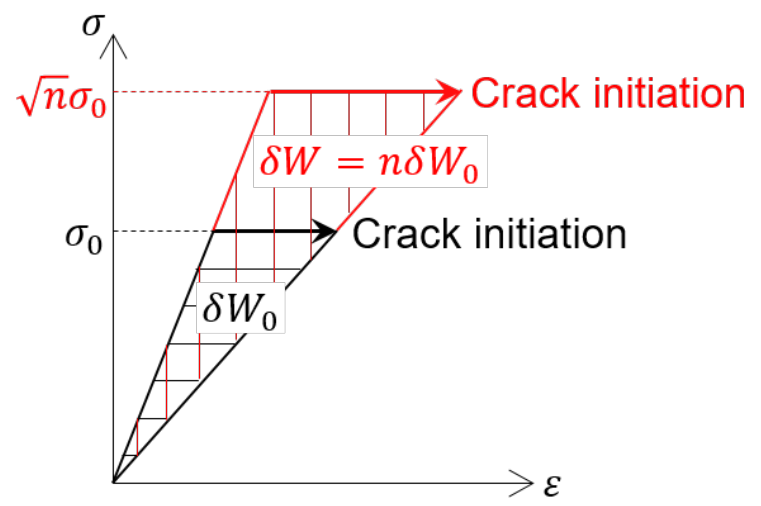

Fig. 9 Amount of work and applied stress required for crack generation. 


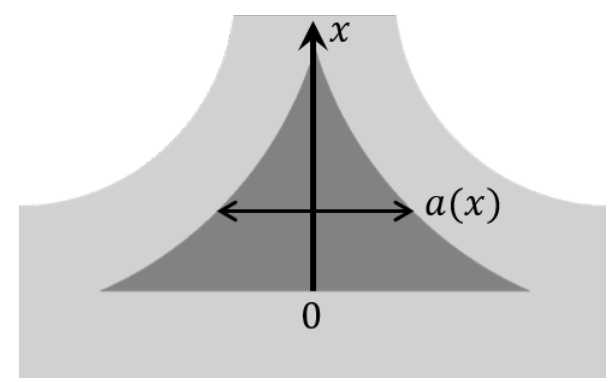

Fig. $10 \mathrm{X}$ axis and width of $90^{\circ}$ layer in the deltoid

(a)

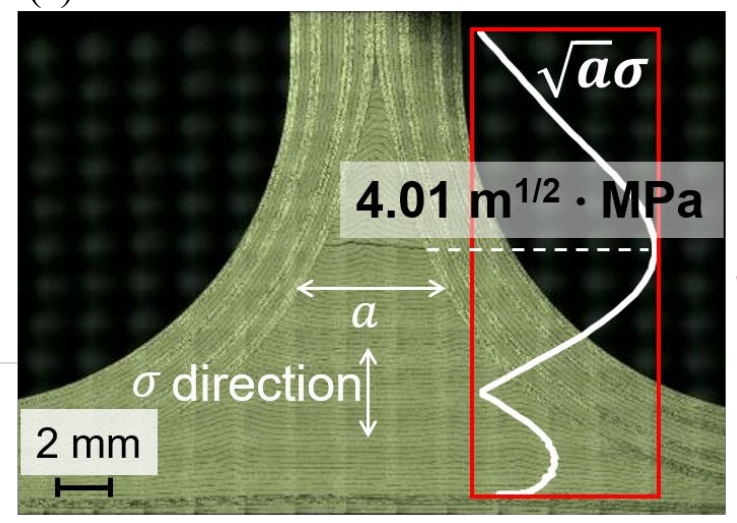

(b)

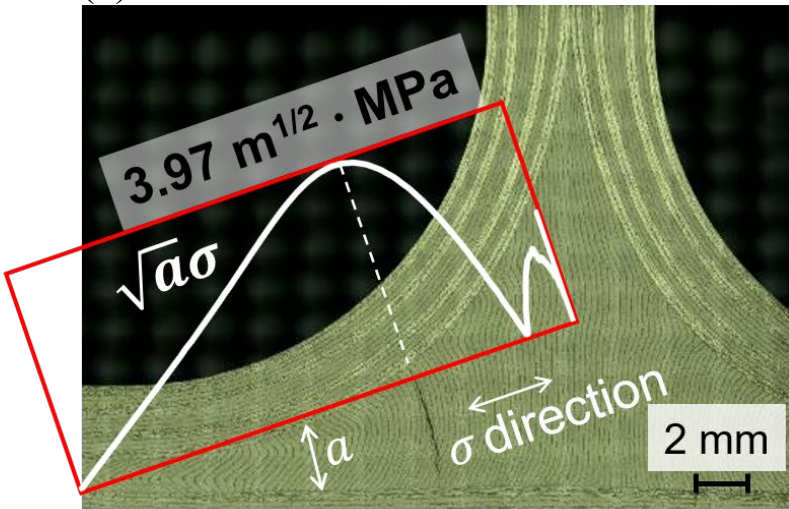

Fig. 11 Comparison of energy-based failure index results and actual failure location.

(a) Horizontally aligned deltoid specimen. (b) Vertically aligned deltoid specimen.

Figure 11(a) shows the comparison of the $\sqrt{a(x)} \sigma(x)$ values derived from the FEA result and the actual failure location in the horizontally aligned deltoid specimen. The location associated with the maximum index value matched well with the failure location in the experiment. The same evaluation was conducted for the vertically aligned deltoid specimen and the result was shown in Fig. 11(b). In the vertically aligned deltoid specimen, the $x$ axis is taken in the normal direction to the lower left corner because the process-induced failure occurred perpendicular to this axis. As with the horizontally aligned deltoid specimen, the location associated with the maximum index value matched well with the failure location. In addition, the maximum value of $\sqrt{a(x)} \sigma(x)$ was nearly the same for these two specimens, i.e., $4.01 \mathrm{~m}^{1 / 2} \cdot \mathrm{MPa}$ for the horizontally aligned deltoid specimen, and $3.97 \mathrm{~m}^{1 / 2} \cdot \mathrm{MPa}$ for the vertically aligned deltoid specimen. These results confirm that process-induced failure of the deltoid of a composite T-joint occurs when the energy-based requirement is met, and that the effects of the width of the $90^{\circ}$ layer should be considered for failure prediction.

\section{Conclusions}

In this study, in-situ strain measurements of the deltoid during curing were conducted using optical fibers. The chemical cure shrinkage strain and thermal shrinkage strain within the deltoid were successfully measured using proposed optical fiber embedment method. From the experiments and FEA supported by the strain measurements with optical fibers, it was confirmed that the process-induced failure of the deltoid of a composite T-joint occurs when the energybased requirement is met. Based on these results obtained from process monitoring with optical 
fiber, it will be possible to devise a method to suppress a process-induced failure of a composite T-joint.

\section{References}

[1] J. Chen, D. Fox, Numerical investigation into multi-delamination failure of composite Tpiece specimens under mixed mode loading using a modified cohesive model, Compos. Struct. 94 (2012) 2010-2016. https://doi.org/10.1016/j.compstruct.2011.12.030

[2] B. Lee, Review of the present status of optical fiber sensors, Opt. Fiber Technol. 9 (2003) 57-79. https://doi.org/10.1016/S1068-5200(02)00527-8

[3] S.T. Kreger, D.K. Gifford, M.E. Froggatt, B.J. Soller, M.S. Wolfe, High resolution distributed strain or temperature measurements in single- and multi-mode fiber using sweptwavelength interferometry, In: Optical Fiber Sensors, Optical Society of America Technical Digest. (2006) Paper ThE42. https://doi.org/10.1364/OFS.2006.ThE42

[4] S. Niwa, K. Takagaki, S. Minakuchi, N. Takeda, Cure shrinkage monitoring and process analysis of CFRP laminates with interlaminar-resin layers, J. Jpn. Soc. Compos. Mater. (in Japanese) 41 (2015) 168-175. https://doi.org/10.6089/jscm.41.168

[5] T.A. Bogetti, J.W. Gillespie, Process-induced stress and deformation in thick-section thermoset composite laminates, J. Compos. Mater. 26 (1992) 626-660. https://doi.org/10.1177/002199839202600502

[6] S. Minakuchi, S. Niwa, K. Takagaki, N. Takeda, Composite cure simulation scheme fully integrating internal strain measurement, Composites Part A 84 (2016) 53-63. https://doi.org/10.1016/j.compositesa.2016.01.001

[7] R. Higuchi, T. Yokozeki, T. Nagashima, T. Aoki, Evaluation of mechanical properties of noncircular carbon fiber reinforced plastics by using XFEM-based computational $\begin{array}{llllll}\text { micromechanics, } & \text { Composites } & \text { Part } & \text { A } & 126 & \text { (2019) }\end{array}$ https://doi.org/10.1016/j.compositesa.2019.105556

[8] S. Seki et al., Evaluation of fatigue life of thick CFRP laminates with toughened interlaminar layers in out-of-plane and in-plane transverse directions, Transactions of the JSME (in Japanese) 83 (2017) 16-00571-16-00571. https://doi.org/10.1299/transjsme.16-00571

[9] A. Parvizi, K.W. Garrett, J.E. Bailey, Constrained cracking in glass fibre-reinforced epoxy cross-ply laminates, J. Mater. Sci. 13 (1978) 195-201. https://doi.org/10.1007/BF00739291 [10] P.P. Camanho, C.G. Davila, S.T. Pinho, L. Iannucci, P. Robinson, Prediction of in situ strengths and matrix cracking in composites under transverse tension and in-plane shear, Composites Part A 37 (2006) 165-176. https://doi.org/10.1016/j.compositesa.2005.04.023 